\title{
Increased carbohydrate and energy consumption in people with Multiple Sclerosis compared to healthy controls
}

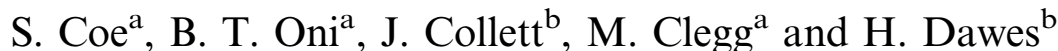 \\ ${ }^{\mathrm{a}}$ Functional Food Centre and ${ }^{\mathrm{b}}$ Movement Science Group, Department of Sport and Health Sciences, Oxford Brookes \\ University, Gipsy Lane, Headington, Oxford OX3 OBP.
}

Multiple sclerosis (MS) is a disease of the central nervous system involving an autoimmune attack on the myelin sheath coating nerve fibres. Studies have shown that people with MS are at a greater risk of malnutrition, being more likely to be both underweight and overweight compared to the general public ${ }^{(1)}$. Also, numerous lifestyle factors including diet have been shown to affect certain symptoms associated with the disease. Therefore quality of life may be improved through dietary changes in people with MS; however few studies have looked at the diet quality and specific nutrient intake in this population.

The objective of this preliminary study was to assess and compare the dietary pattern of 10 MS patients and 10 healthy controls. Body composition was measured in both groups to determine potential malnutrition.

The Diet History Questionnaire was used to assess nutrient intake ${ }^{(2)}$, and body mass index (BMI; $\mathrm{kg} / \mathrm{m}^{2}$ ) was evaluated against the international classification according to the World Health Organisation (WHO) ${ }^{(3)}$.

Table 1. BMI values, and energy and macronutrient consumption in control and MS subjects.

\begin{tabular}{|c|c|c|c|c|c|}
\hline & Control group & SD & MS group & SD & $p$-value \\
\hline BMI $\left(\mathrm{kg} / \mathrm{m}^{2}\right)$ & $26 \cdot 72$ & $4 \cdot 16$ & $25 \cdot 43$ & 3.68 & 0.47 \\
\hline Energy (kcal) & 1582.91 & 397.06 & $2090 \cdot 05$ & $548 \cdot 45$ & 0.03 \\
\hline Carbohydrate $(\mathrm{g})$ & 202.79 & $64 \cdot 55$ & $279 \cdot 22$ & $74 \cdot 88$ & $0 \cdot 03$ \\
\hline Fat $(\mathrm{g})$ & $56 \cdot 63$ & $22 \cdot 35$ & $71 \cdot 26$ & $26 \cdot 89$ & $0 \cdot 20$ \\
\hline Protein $(\mathrm{g})$ & $61 \cdot 65$ & $15 \cdot 59$ & $67 \cdot 34$ & $24 \cdot 45$ & $0 \cdot 54$ \\
\hline Fibre $(\mathrm{g})$ & 17.97 & $8 \cdot 27$ & $22 \cdot 77$ & 8.93 & 0.23 \\
\hline
\end{tabular}

$\mathrm{SD}=$ standard deviation

The energy intake in the MS group was significantly higher than the control $(p<0.05)$ and this was highlighted further when correcting for body mass $(\mathrm{kg})$. However, BMI was not significantly different between the two groups. Total carbohydrate intake $(\mathrm{g})$ was significantly higher in the MS group $(p<0.05)$ with percentage of carbohydrate above the DRV ${ }^{(4)}$ and fat and protein below. This increase in carbohydrate was highlighted by an increase in fructose, glucose, sucrose and total sugars $(p<0.05)$ in the MS group compared to the control group. There was no difference in micronutrients, fibre or food group consumption.

Differences exist between intakes of certain nutrients in people with MS compared to controls. Although energy and carbohydrate intake were greater in MS subjects compared to controls, there was no difference in BMI between the groups. However this preliminary work used a small sample size and future studies will be aimed at assessing a larger sample to determine the relationship between dietary intake, specifically energy and carbohydrate intake, energy expenditure and body composition in people with MS.

1. Payne A (2001) J Hum Nutr Dietet 14, 349-357.

2. Continuing survey of food intakes by individuals (1998) Uni States Depart Agric.

3. WHO (2012) BMI Classification.

4. Whitton C et al. (2011) Br J Nutr 106, 1899-1914. 